\title{
Schwachsinnig und abartig? Wahrgenommene Stigmatisierung und tatsächliche Etikettierungseffekte der alten und modernisierten Eingangsmerkmale in $\S 20$ Strafgesetzbuch (StGB)
}

\author{
D. Rösch · S. Ruckelshaußen ${ }^{1}$ J. Kirsch ${ }^{1}$ S. Gerhards ${ }^{1}$ L. A. Sroka' · R. Imhoff' \\ Eingegangen: 8. Oktober 2020 / Angenommen: 9. November 2020 / Online publiziert: 8. Dezember 2020 \\ (c) Der/die Autor(en) 2020
}

\section{Zusammenfassung}

Bei der Beurteilung der Schuldfähigkeit eines Beschuldigten nach §§ 20, 21 StGB muss zunächst beurteilt werden, ob ein Eingangsmerkmal vorliegt, das verminderte oder reduzierte Schuldfähigkeit bedingen könnte, sowie ob dieses Eingangsmerkmal den Beschuldigten hinreichend in Einsichts- und/oder Steuerungsfähigkeit beeinflusst. Die bislang geltenden Eingangsmerkmale sind z.T. als veraltet und stigmatisierend kritisiert worden - insbesondere die der schweren anderen seelischen Abartigkeit und des Schwachsinns -, sodass im Jahr 2019 ein Gesetz zur Änderung des Strafgesetzbuches vorgelegt wurde, das eine Modernisierung der Begriffe beinhaltet. Die vorliegende Studie überprüft erstmals, ob die verschiedenen Begriffe (Intelligenzminderung vs. Schwachsinn; seelische Störung vs. seelische Abartigkeit) als unterschiedlich stigmatisierend wahrgenommen werden und zu unterschiedlicher Personenwahrnehmung führen. Zu diesem Zweck füllten 208 Personen, darunter rechtspsychologische Laien $(n=131)$ und Personen mit Vorwissen $(n=77)$, Onlinefragebogen aus, in denen sie sowohl anhand von Fallvignetten u.a. Schuldfähigkeit, Gefährlichkeit und Einsichtsfähigkeit von Personen einschätzen mussten als auch die unterschiedlichen Eingangsmerkmale direkt beurteilten. Die Ergebnisse zeigen, dass die überarbeiteten Begriffe als signifikant juristisch angemessener, positiver konnotiert, weniger stigmatisierend und weniger abwertend eingestuft werden. Auch bei einer qualitativen Untersuchung von Assoziationen der Probanden zu den jeweiligen Begriffen zeigten sich deutlich negativere Konnotationen der alten Begriffe. Gleichzeitig zeigte sich jedoch, dass sich die Bewertung von Individuen, die jeweils mit diesen Begriffen betitelt wurden, nicht unterschied, auch nicht bei rechtspsychologischen Laien. Sowohl rechtspsychologische Laien als auch Personen mit Vorwissen haben demnach zwar eindeutige Ansichten zu den vorliegenden Begriffen, diese wirken sich jedoch nicht signifikant auf ihre Personenwahrnehmung aus.

Schlüsselwörter Schuldfähigkeit $\cdot$ Begutachtung $\cdot$ Personenwahrnehmung $\cdot$ Stigmatisierung $\cdot$ Regierungsentwurf

Zum Zwecke der besseren Lesbarkeit wird auf die unterschiedliche geschlechtsspezifische Schreibweise verzichtet. Die gewählte männliche Form ist in diesem Sinne geschlechtsneutral zu verstehen.

Zusatzmaterial online Zusätzliche Informationen sind in der Online-Version dieses Artikels (https://doi.org/10.1007/s11757020-00640-x) enthalten.

Prof. Dr. R. Imhoff

roland.imhoff@uni-mainz.de

1 Institut für Psychologie, Abteilung Sozial- und

Rechtspsychologie, Johannes Gutenberg-Universität Mainz,

Binger-Str. 14-16, 55122 Mainz, Deutschland 


\title{
Moronic and abnormal? Perceived stigmatization and actual labelling effects of the old and modernized entry characteristics in \$20 German Penal Code
}

\begin{abstract}
According to German law, assessing the culpability of an accused person requires an initial assessment of whether the accused suffers from a condition that might in principle reduce or diminish culpability. This is followed by an evaluation of whether this condition did indeed impair the ability to either understand or control their actions. The impairing condition has to fall into one of four predefined classes, some which have been criticized for their outdated and stigmatizing terminology (in particular, "schwere andere seelische Abartigkeit" and "Schwachsinn"). In 2019, a law to amend the penal code and modernize the terminology was proposed. The present study produces the first empirical investigation of whether the previous and modernized terms (Intelligenzminderung vs. Schwachsinn; seelische Störung vs. seelische Abartigkeit) are perceived as differing in stigmatizing and lead to different perceptions of persons. For this purpose, 208 persons, including lay people in legal psychology $(n=131)$ and persons with expertise $(n=77)$, completed online questionnaires in which they had to assess, among other things, the culpability, dangerousness and insightfulness of persons on the basis of case vignettes and directly classify the terms. The results show that the revised terms are perceived as more legally appropriate, more positively connotated, less stigmatizing and less pejorative. A qualitative investigation of the respondents' associations with the terms also revealed more negative connotations of the old terms. At the same time, however, it was shown that the evaluation of individuals who were titled with these terms did not differ, even among lay persons. Both lay people and persons with expertise therefore have strong opinions on the terms but these do not have a significant effect on their perception of individuals.
\end{abstract}

Keywords Culpability $\cdot$ Expert testimony $\cdot$ Person perception $\cdot$ Stigmatization $\cdot$ Draft bill

\section{Einleitung}

Die Feststellung der Schuldfähigkeit wird im deutschen Strafrecht über die Paragraphen $\$ 20$ und $\$ 21$ StGB geregelt. Grundsätzlich geht das Gesetz davon aus, dass erwachsene Menschen in der Regel schuldfähig sind, Abweichungen müssen geprüft werden (Schneider, Frister, \& Olzen 2020). Die Feststellung der Schuldunfähigkeit bezieht sich dabei immer nur auf eine konkrete Situation eines Menschen, sie kann somit nicht generalisiert und auf andere Situationen übertragen werden (Nedopil 2007). Bei der Prüfung der Schuldunfähigkeit wird in einem ersten Schritt ermittelt, ob bei einer Person zum Zeitpunkt der Tat eine Einschränkung vorlag, die sich unter einem der in \$20 StGB genannten Eingangsmerkmale subsumieren lässt. Es handelt sich nach Gesetzestext um die „tiefgreifende Bewusstseinsstörung“, die „krankhafte seelische Störung“, die „schwere andere seelische Abartigkeit“ und den „Schwachsinn“. In einem zweiten Schritt muss anschließend geprüft werden, ob die psychische Störung zum Zeitpunkt der Tat dazu führte, dass der Täter nicht fähig war, das Unrecht der Tat einzusehen (Einsichtsfähigkeit) oder nach dieser Einsicht zu handeln (Steuerungsfähigkeit) (Nedopil 2007; Schneider et al. 2020). Somit bedeutet eine Diagnose nach den Klassifikationssystemen allein nicht direkt, dass Schuldunfähigkeit vorliegt, sie weist jedoch auf eine nichtgeringfügige Beeinträchtigung hin (Theune 2002).

Aus heutiger Sicht sind insbesondere Begriffe wie „Schwachsinn“ oder „,seelische Abartigkeit“ ungebräuch- lich, stark negativ konnotiert und damit nicht mehr zeitgemäß. Die Begriffe wurden im Rahmen des 2. Strafrechtsreformgesetzes 1969 eingeführt und orientierten sich am Krankheitsbegriff Kurt Schneiders (Bundesministerium der Justiz und für Verbraucherschutz, BMJV 2020). Laut Schneider lassen sich psychopathologische Diagnosen in krankhafte und nichtkrankhafte, d.h. nicht auf organische Ursachen rückführbare, Diagnosen unterteilen (Schneider 2007). Schon damals galt dieser Krankheitsbegriff als umstritten und war nicht in der gesamten Medizin anerkannt. Er wurde jedoch aus juristischen Gründen für die Paragraphen gewählt, da er den engsten und schärfsten Krankheitsbegriff darstellte (Krümpelmann 1976). Heute gilt Schneiders Krankheitsbegriff eindeutig als überholt, und auch die gebräuchlichen Klassifikationssysteme für psychiatrische Diagnosen orientieren sich nicht an ihm (Schiemann 2019), denn hier wird durchgängig von „Störungen“ gesprochen. Dennoch sind die $\S \S 20$ und 21 weiterhin sowohl in Wortwahl als auch Systematik an ihm orientiert; die Subsumption muss sich also weiterhin danach ausrichten.

Der Begriff des Schwachsinns war in den 1970er-Jahren in der Medizin noch gebräuchlich, ist jedoch mittlerweile aus medizinischer sowie psychologischer Sicht veraltet und wird aufgrund seiner ,historisch und umgangssprachlich bedingten sozial diskriminierenden Tönung " in der Medizin seit Jahren nicht mehr verwendet (Wegener 1989, S. 320). Mit dem Begriff der Abartigkeit verhält es sich jedoch anders. Dieser war bereits zum Zeitpunkt der Einführung 
1975 aufgrund seines Bezugs zum Nationalsozialismus und zu Musterungsbestimmungen der Wehrmacht stark umstritten (Wegener 1989; Janßen 1994). Laut Wegener (1989) hat ,der Gesetzgeber [...] einen historisch belasteten Begriff verwendet“ (S. 325). Er fasst zusammen: „Man fragt sich, wie es möglich war, einen Begriff mit solcher Vergangenheit aufzunehmen, welcher dazu noch in jener Zeit als Schimpfwort in Gebrauch war?“" (S. 317). Zusätzlich zu diesem historischen Hintergrund empfahl bereits Schneider selbst, den Psychopathiebegriff im juristischen Kontext zu vermeiden, der lange Zeit in enger Verbindung mit der seelischen Abartigkeit stand. Grund hierfür war, dass die von Schneider ,strikt betonte Wertneutralität dieser Diagnose in praxi nicht aufrecht zu erhalten war" (Krümpelmann 1976; Meyer 1976, S. 53).

Im Laufe der Jahre wurde vermehrt die diskriminierende und stigmatisierende Wirkung der Begriffe bemängelt und eine Überarbeitung gefordert (z. B. Meyer 1976; Janßen 1994; Wegener 1989; Schiemann 2019). Auch der Referentenentwurf zur Änderung des Strafgesetzbuches, der am 04.09.2019 vorgelegt wurde, räumt dies ein. Hier wird genannt, dass die Begriffe Schwachsinn und Abartigkeit „zunehmend als stigmatisierend oder gar diffamierend“ angesehen werden und teilweise deren Abschaffung gefordert wird (BMJV 2019, S. 24). Der 2019 vorgelegte Entwurf zur Gesetzesänderung sieht eine Änderung des Eingangsmerkmals Schwachsinn in „Intelligenzminderung“ vor sowie die Änderung der schweren anderen seelischen Abartigkeit in die ,schwere andere seelische Störung“.

Obwohl es also zahlreiche Stimmen gibt, die die alten Begriffe als stigmatisierend kritisieren und deren Novellierung fordern, ist diese Debatte bis dato unerwartet frei von Empirie. Ob ein Begriff stigmatisierend ist in dem Sinne, dass er als negativ empfunden wird und die Wahrnehmung einer Person negativ einfärbt, ist aus Sicht der Sozialwissenschaften eine empirische Frage. Die vorliegende Studie überprüft deshalb erstmals, ob die veralteten Begriffe des Schwachsinns und der schweren anderen seelischen Abartigkeit als stigmatisierend empfunden werden, und ob sie zu unterschiedlicher Personenwahrnehmung führen.

Letzteres ist insbesondere relevant, da, unabhängig von einer normativ-ethischen Diskussion um die Angemessenheit unterschiedlicher Begrifflichkeiten, Sprache Realitäten schaffen kann. So geht der modifizierte Labeling-Ansatz (Link et al. 1989) davon aus, dass das Label über die eigentliche Symptomatologie hinaus, negative Effekte haben kann. Dies gilt insbesondere auch für die Fremdwahrnehmung. Eine identisch beschriebene Person wird z.T. als aggressiver, gefährlicher, weniger vertrauenswürdig und kompetent eingestuft, wenn dieser Beschreibung das diagnostische Label „Schizophrenie“ hinzugefügt wird (Imhoff 2016). Ebenso macht es einen messbaren Unterschied, ob Menschen als ,,pädophil“ oder aber ,,sexuell an präpubertä- ren Kindern interessiert" bezeichnet werden, wobei Ersteren mit drastischeren Bestrafungswünschen begegnet wird (Imhoff 2015). Zu dem Grad, zu dem die Eingangsmerkmale an sich also einen nachweisbaren Einfluss auf die Wahrnehmung der mit ihnen bezeichneten Person haben, wäre die Fairness des Verfahrens infrage zu stellen.

So könnte es zu einem Halo-Effekt kommen, unter dem die Tendenz verstanden wird, ,faktisch unabhängige oder nur mäßig korrelierte Eigenschaften von Personen fälschlicherweise als zusammenhängend wahrzunehmen" (Schweizer 2005, S. 230). Die Bezeichnung als schwachsinnig oder abartig führt demnach potenziell zur Assoziation diverser eigentlich hiervon unabhängiger Eigenschaften und somit zum Nachteil der beschuldigten Person.

Die vorliegende Studie soll einen empirischen Beitrag zur stigmatisierenden Wirkung der Begriffe ,schwere andere seelische Abartigkeit" und „Schwachsinn“ auf zwei Ebenen leisten. In einem ersten Schritt soll eruiert werden, inwiefern die Begriffe tatsächlich als stigmatisierend erlebt werden. Hierzu werden direkte Bewertungen herangezogen, ebenso wie offene Nennung von Assoziationen. Die zweite Fragestellung ist der kritischere Test und bezieht sich auf die tatsächliche Wirkung der Begriffe, sowohl bezogen als Schuld- und Einsichtsfähigkeit als auch auf die drei relevanten Dimensionen der Stigmatisierung (Gefährlichkeit, Seltenheit, eigene Verantwortlichkeit; Feldman und Crandall 2007). Hierzu wird in einem experimentellen Ansatz die aus der modifizierten Labeling-Theorie abgeleitete Hypothese überprüft, dass Straftäter als schuldfähiger wahrgenommen werden, wenn die modernisierten Begriffe genutzt werden (Hypothese 1). Zusätzlich bestehen die jeweils auf die spezifischen Eingangsmerkmale bezogenen Hypothesen, dass Straftäter als einsichtsfähiger wahrgenommen werden, wenn der Begriff Intelligenzminderung verwendet wird (im Vergleich zu „Schwachsinn“) (Hypothese 2) und als gefährlicher wahrgenommen werden, wenn der Begriff schwere andere seelische Abartigkeit (im Vergleich zu ,Störung“) verwendet wird (Hypothese 3). Da es Personen, die mit den konkreten Begrifflichkeiten vertraut sind, möglich ist, die tatsächliche Äquivalenz des bezeichneten Eingangsmerkmals zu erkennen, wurde erwartet, dass alle Unterschiede unter Laien ausgeprägter ausfallen als unter Personen mit Vorwissen (Hypothese 4).

$\mathrm{Zu}$ Explorationszwecken wurden zusätzlich die wahrgenommene Sympathie, Therapieempfänglichkeit, Rückfallrisiko sowie Abweichung von den geltenden Normen, Vollbesitz geistiger Kräfte sowie Impulssteuerung beschriebener Täter erhoben. Angedachte explorative Analysen zu Unterschieden zwischen Juristen und Psychologen wurden aufgrund der geringen Anzahl teilnehmender Juristen $(n=7)$ nicht durchgeführt. 


\section{Methode}

\section{Stichprobe}

Die Probanden wurden über universitätsinterne E-Mail-Verteiler der Johannes Gutenberg-Universität Mainz sowie soziale Medien rekrutiert. Insgesamt umfasste die Stichprobe $n=218$ abgeschlossene Fragebogen. Im Einklang mit den in der Präregistrierung ${ }^{1}$ angegebenen Ausschlusskriterien (Abschluss des Fragebogens unter 5 min, unvollständige Fragebogen, Nennung des Studienziels bei der Zwischenüberprüfung) wurden 10 Personen ausgeschlossen. Die endgültige Stichprobe umfasste somit $n=208$ Personen (165 Frauen, 41 Männer, 2 Personen, die sich als divers identifizierten) im Alter von 18 bis 72 Jahren $(M=26,59 ; S D=9)$. Für die Studie wurde eine Selbsteinschätzung des rechtspsychologischen Vorwissens erhoben. Unter den 208 Probanden befanden sich laut eigenen Angaben 131 rechtspsychologische Laien und 77 Personen mit Vorwissen. Ein Großteil der Stichprobe bestand aufgrund der Rekrutierung aus Studierenden $(n=178)$.

\section{Direkte Bewertung ${ }^{2}$}

Zur Messung der Einstellung zu den Begriffen wurden Probanden zunächst offen danach gefragt, was Personen mit den jeweiligen Begriffen assoziieren. Diese Abfrage diente dazu, anhand von Stichworten und Kategorien mittels Word Clouds einen Gesamtüberblick darüber erhalten zu können, ob die unterschiedlichen Begrifflichkeiten mit verschiedenen Ausdrücken und Themen in Verbindung gebracht werden. Anschließend wurde auf einer 10-Punkte-Ratingskala nach Konnotation, abwertender Wirkung, juristischer Angemessenheit und stigmatisierender Wirkung der Begriffe gefragt. Zuletzt wurde in einer direkten Gegenüberstellung für jedes Eingangsmerkmal abgefragt, ob der neue oder modernisierte Begriff als stigmatisierender empfunden wird.

\section{Experimentelle Überprüfung der Wirkung}

\section{Manipulation}

Allen Probanden wurden im ersten Teil der Studie nacheinander 4 Fallvignetten präsentiert, in denen jeweils eine verurteilte Person und ihr Vergehen beschrieben wurden. Probanden sollten auf Basis dieser Texte eine Reihe von Einschätzungen vornehmen (z. B. „Für wie gefährlich halten Sie die beschriebene Person?“ oder „Wie sehr glauben Sie, kann die beschriebene Person die Folgen ihrer

\footnotetext{
1 Präregistrierung abrufbar unter: https://aspredicted.org/3ji7r.pdf.

2 Die komplette Studie sowie der finale Datensatz abrufbar unter: https://osf.io/bsdzk.
}

Handlung absehen?"). Die Fallvignetten waren inhaltlich angelehnt an Urteile des Bundesgerichtshof (BGH) sowie eine Fallstudie der Deutschen Heilpädagogischen Gesellschaft (DHG, 2013; Rechtsanwaltskanzlei Strate und Ventzke 2020a, 2020b, 2020c) $)^{3}$. Die Fälle wurden stark gekürzt und enthielten neben einer kurzen klinischen sowie strafrechtlichen Vorgeschichte der beschriebenen Personen Informationen zur Tat sowie eine kurze klinische Beschreibung, die zur Einschätzung der jeweiligen Eingangsmerkmale durch die Sachverständigen führten. Die Fälle wurden parallelisiert, indem die Delikte in allen Fallvignetten Körperverletzung oder Diebstahl darstellten. Jeweils 2 der 4 Fälle befassten sich mit Personen, die durch einen forensisch-psychiatrischen Sachverständigen Schwachsinn bzw. Intelligenzminderung bescheinigt bekamen, sowie 2 Fälle, in denen die Beschriebenen eine schwere andere seelische Abartigkeit (SASA)/Störung (SASS) bescheinigt bekamen. Es wurde randomisiert zugeordnet, welche Bezeichnung die Fallvignetten enthielten. Während die Begriffe der SASA bzw. SASS als Zwischensubjektfaktor manipuliert wurden, sodass Probanden jeweils den einen oder anderen Begriff in beiden Vignetten präsentiert bekamen, wurde jedem Probanden aufgrund eines Programmierungsfehlers bei der Randomisierung jeweils eine Vignette mit dem Begriff „Schwachsinn“ und „Intelligenzminderung“ präsentiert (die Begriffe wurden hier also als Innersubjektfaktor realisiert).

\section{Abhängige Variablen}

Als abhängige Variablen wurden im Rahmen dieser Studie Schuldfähigkeit, Einsichtsfähigkeit sowie Gefährlichkeit betrachtet. Schuldfähigkeit ergibt sich direkt aus dem Item: „Für wie schuldfähig für seine Tat halten Sie die beschriebene Person?" Die Variablen Einsichtsfähigkeit und Gefährlichkeit wurden mittels Skalenbildung erstellt. Unter Einsichtsfähigkeit wird im Sinne des §20 StGB die Fähigkeit verstanden, das Unrecht der eigenen Tat einzusehen. Es wurde hierzu eine Skala über Mittelung der Itemwerte der Items: „Wie sehr ist die beschriebene Person Ihrer Meinung nach dazu fähig, das Unrecht ihrer Tat einzusehen?“ „Wie sehr, glauben Sie, kann die beschriebene Person abstrakte Regeln verstehen?“ sowie „Wie sehr glauben Sie, kann die beschriebene Person die Folgen ihrer Handlung absehen?" gebildet. Einsichtsfähigkeit ergab somit eine 7-Punkte-Skala, auf der höhere Werte höhere Einsichtsfähigkeit bedeuten. Für die Skala Gefährlichkeit wurden die Itemwerte der Items: „Für wie gefährlich halten Sie die beschriebe-

\footnotetext{
3 Originalfälle abrufbar unter: https://www.hrr-strafrecht.de/hrr/5/10/ 5-240-10.php?referer=db; https://www.hrr-strafrecht.de/hrr/2/18/2112-18.php; https://www.hrr-strafrecht.de/hrr/1/03/1-346-03.php3? referer=db; http://dhg-kontakt.de/wp-content/uploads/2015/12/DHGSchrift-18.pdf.
} 
ne Person?“ und „Als wie stark ausgeprägt schätzen Sie das Rückfallrisiko der beschriebenen Person ein?" gemittelt. Hier ergab sich ebenfalls eine 7-Punkte-Skala, auf der höhere Werte höhere Gefährlichkeit bedeuten.

\section{Ablauf}

Nach der Begrüßung bearbeiteten die Teilnehmenden zuerst die Fallvignetten zur experimentellen Überprüfung der Wirkung, bevor sie im direkten Anschluss gefragt wurden, ob sie eine Vermutung bezüglich des Ziels der Studie hatten. Erst anschließend wurden sie direkt zu der stigmatisierenden Konnotation der Begriffe befragt. Diese Reihenfolge wurde gewählt, damit die experimentelle Überprüfung der tatsächlichen stigmatisierenden Wirkung unbeeinflusst vom Wissen über das Ziel der Studie blieb. Im Folgenden wird, von der Chronologie der Studie abweichend, zuerst die Bewertung der Begriffe und anschließend ihre Wirkung berichtet.

\section{Ergebnisse}

\section{Direkte Bewertung der Begrifflichkeiten}

Übereinstimmend mit der Hypothese zeigt sich deutlich, dass sowohl im Falle der SASA/SASS als auch im Falle Schwachsinn/Intelligenzminderung die alten Begriffe jeweils als signifikant abwertender, stigmatisierender, negativer konnotiert und weniger juristisch angemessen beurteilt wurden als die modernisierten Begriffe (Tab. 1 und 2).
In der direkten Gegenüberstellung zeigte sich noch einmal deutlich, dass im Falle beider Eingangsmerkmale die alten Begriffe als stigmatisierender bzw. negativer wahrgenommen wurden (Abb. 1).

Neben der direkten Abfrage zur Stigmatisierung der Begriffe konnten die Probanden in einem freien Textfeld ihre Assoziationen zu den einzelnen Begriffen eintragen. In der qualitativen Analyse dieses freien Antwortformats wurden die Assoziationen zunächst zu Stichworten gekürzt und nach inhaltlichen Kategorien sortiert (z. B. wurden die Antworten ,,alter Ausdruck“, ,,veraltet“, ,,altmodisch“, „nicht mehr zeitgemäß“ usw. zusammengefasst). Durch die Kategorien konnte zum Zwecke der Übersichtlichkeit sichergestellt werden, dass keine genannten Assoziationen verloren gingen. Anschließend wurden die Begriffe nach der Häufigkeit ihrer Nennung geordnet und aus den gebildeten Kategorien Word Clouds angelegt (Zusatzmaterial online: Abb. A1-A4), um die Unterschiede in den Assoziationen und die Häufigkeit der Nennung der Begriffe zu veranschaulichen. Dem Zusatzmaterial kann die entsprechende Word Cloud zu jedem Eignungsmerkmal entnommen werden. (Aus Darstellungsgründen werden die Begriffe, die nur einmal genannt wurden, nicht dargestellt.) In Tab. 3 sind die 10 am häufigsten genannten Assoziationen je Eingangsmerkmal aufgelistet. Wie bereits in der direkten Gegenüberstellung der Begriffe ersichtlich, zeigt sich auch hier, dass mit den alten Begriffen eine negativere Konnotation einhergeht. Insbesondere bei der Gegenüberstellung der Begriffe der SASA und SASS wird dies deutlich: Mit der alten SASA wurden von den Probanden im Vergleich zur neuen SASS eher Begriffe assoziiert, die die beschriebene Person als unmenschliche, zur Vergewaltigung fähige und gefähr-

Tab. 1 Ergebnisse des t-Tests für abhängige Stichproben für den Vergleich der Bewertung der Begriffe „SASA“ und „SASS“

\begin{tabular}{|c|c|c|c|c|c|c|c|}
\hline & \multicolumn{3}{|c|}{ SASS } & \multirow{2}{*}{$\begin{array}{l}\text { SASA } \\
S D\end{array}$} & \multirow[b]{2}{*}{$t(207)$} & \multirow[b]{2}{*}{$p$} & \multirow[b]{2}{*}{$d$} \\
\hline & $\bar{M}$ & $S D$ & $M$ & & & & \\
\hline$\overline{\text { Abwertung }}$ & 4,46 & 2,59 & 8,38 & 2,25 & 20,160 & $<0,001$ & 2,802 \\
\hline Stigmatisierung & 5,93 & 2,47 & 8,51 & 2,18 & 14,566 & $<0,001$ & 2,025 \\
\hline Konnotation & 4,24 & 1,67 & 1,60 & 1,21 & $-22,067$ & $<0,001$ & $-3,068$ \\
\hline Juristische Angemessenheit & 7,05 & 2,47 & 3,08 & 2,66 & $-18,444$ & $<0,001$ & $-2,564$ \\
\hline
\end{tabular}

Anmerkung. Bei „Abwertung“, „Stigmatisierung“ und ,juristischer Angemessenheit“ stehen höhere Mittelwerte für eine stärkere Bewertung in diese Richtung. Bei „Konnotation“ stehen höhere Mittelwerte für eine positivere Konnotation

Tab. 2 Ergebnisse des t-Tests für abhängige Stichproben für den Vergleich der Bewertung der Begriffe „Intelligenzminderung“ und „Schwachsinn“

\begin{tabular}{|c|c|c|c|c|c|c|c|}
\hline & \multicolumn{2}{|c|}{ Intelligenzminderung } & \multicolumn{2}{|c|}{ Schwachsinn } & \multirow[b]{2}{*}{$t(207)$} & \multirow[b]{2}{*}{$p$} & \multirow[b]{2}{*}{$d$} \\
\hline & $M$ & $S D$ & $\bar{M}$ & $S D$ & & & \\
\hline Abwertung & 4,23 & 2,40 & 8,38 & 2,12 & 20,173 & $<0,001$ & 2,804 \\
\hline Stigmatisierung & 6,03 & 2,38 & 8,25 & 2,32 & 10,918 & $<0,001$ & 1,518 \\
\hline Konnotation & 4,46 & 1,62 & 2,30 & 1,59 & $-13,773$ & $<0,001$ & $-1,915$ \\
\hline Juristische Angemessenheit & 7,90 & 2,04 & 2,46 & 2,25 & $-26,282$ & $<0,001$ & $-3,653$ \\
\hline
\end{tabular}

Anmerkung. Bei „Abwertung“, „Stigmatisierung“ und ,juristischer Angemessenheit“ stehen höhere Mittelwerte für eine stärkere Bewertung in diese Richtung. Bei „Konnotation“ stehen höhere Mittelwerte für eine positivere Konnotation 
Abb. 1 Direkte Gegenüberstellung bei der Frage ,Welchen Begriff halten Sie für negativer/stigmatisierender?" für Schwachsinn vs. Intelligenzminderung bzw. SASA vs. SASS $; n=208$

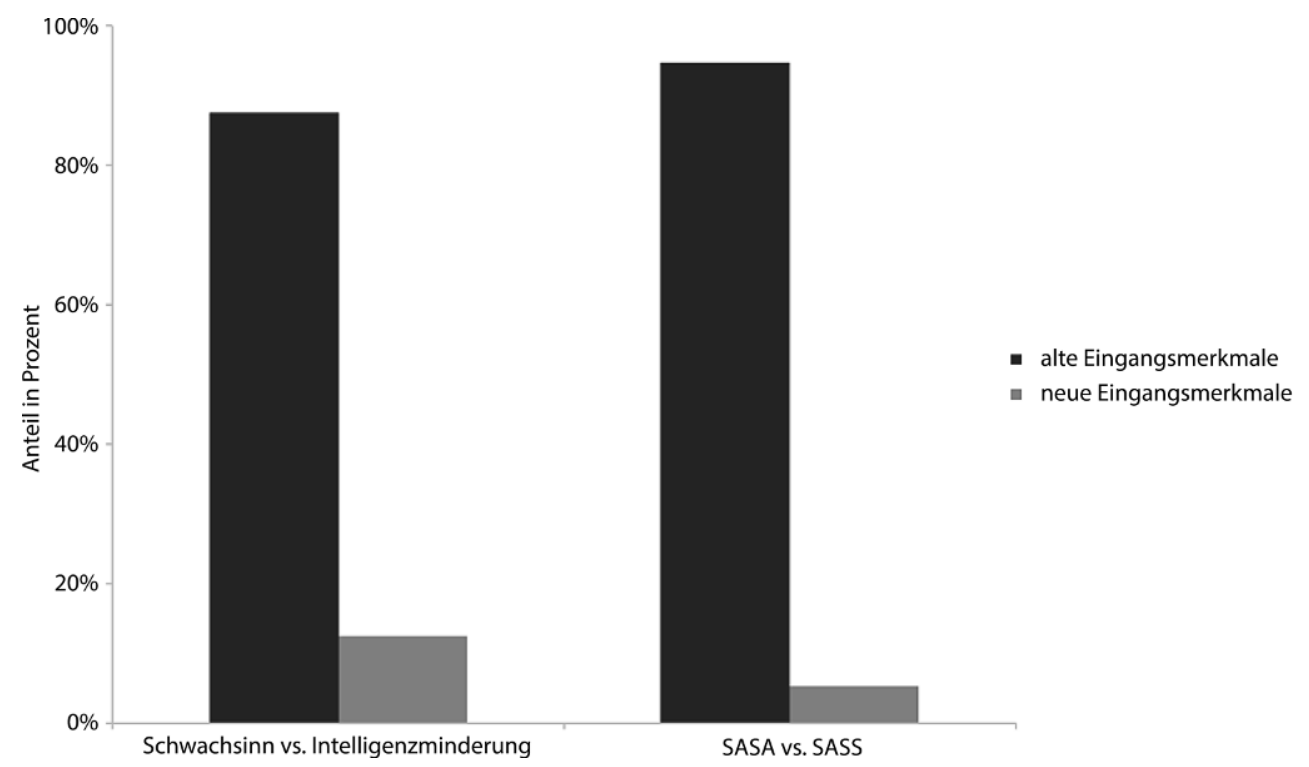

liche Person darstellen. Unter anderem die Begriffe bösartig, grauenvoll und Ekel werden genannt. Dagegen wurden mit SASS eher spezifische psychiatrische Krankheitsbilder, Leidensdruck oder traumatische Erfahrungen der beschriebenen Person assoziiert.

\section{Tatsächlich stigmatisierende Wirkung}

Ausgehend von dieser deutlichen negativen Bewertung der Begriffe war die kritische Frage, ob die Begriffe tatsächlich unterschiedliche Personenwahrnehmung nach sich zogen abhängig vom Vorwissen der Probanden. Dazu wurden getrennt für die beiden Eingangsmerkmale separate 2 (Begriff: alt vs. neu) $\times 2$ (Vorwissen: Laie vs. Vorwissen) ANOVA für jede abhängige Variable durchgeführt (Tab. 4, 5, 6 und 7).

Entgegen der Hypothese hatte die Verwendung des Begriffes SASA oder SASS keinen signifikanten Einfluss auf die Einschätzung der Schuldfähigkeit. Die einzigen systematischen Zusammenhänge zeigten sich mit dem Vorwis- sen, insofern, als dass rechtspsychologisch oder juristisch vorgebildete Teilnehmer deskriptiv insgesamt ein geringeres $\mathrm{Ma} ß$ an Schuldfähigkeit, ein höheres $\mathrm{Ma}$ an Einsichtsfähigkeit und ein geringeres $\mathrm{Ma}$ an Gefährlichkeit annahmen. Für die zusätzlichen - explorativ erhobenen - Variablen Sympathie, Therapieempfänglichkeit, Rückfallrisiko sowie Abweichung von den geltenden Normen, ergaben sich für das Eingangsmerkmal SASA/SASS ebenfalls keine Effekte (Zusatzmaterial online: Tabelle A5). Es gab jedoch signifikante Unterschiede in der Einschätzung des Vollbesitzes der geistigen Kräfte, $t(186,16)=-2,49, p=0,014$, $d=-0,352$, sowie der Einschätzung der Impulssteuerung, $t(206)=2,42, p=0,016, d=0,337$. Für den Vollbesitz geistiger Kräfte fielen die Einschätzungen für den alten Begriff der SASA höher aus $(M=4,07, S D=1,02)$ als für den Begriff der SASS $(M=3,67, S D=1,25)$. Impulssteuerung wurde für den alten Begriff der SASA geringer eingeschätzt $(M=5,26, S D=0,91)$ als für den Begriff der SASS $(M=5,58, S D=0,97)$.

Tab. 3 Die 10 am häufigsten genannten Assoziationen je Eingangsmerkmal

\begin{tabular}{|c|c|c|c|c|}
\hline & $\begin{array}{l}\text { Schwere andere seelische } \\
\text { Abartigkeit }\end{array}$ & $\begin{array}{l}\text { Schwere andere seelische } \\
\text { Störung }\end{array}$ & Schwachsinn & Intelligenzminderung \\
\hline 1 & Persönlichkeitsstörung & Psychische Störung & Geminderte Intelligenz & Niedriger Intelligenzquotient (IQ) \\
\hline 2 & Abweichung von der Norm & Trauma & Geistige Behinderung & Geminderte Intelligenz \\
\hline 3 & Psychische Störung & Depression & Dumm/Dummheit & Geistige Behinderung \\
\hline 4 & Abwertend & Persönlichkeitsstörung & Veraltet & Dummheit \\
\hline 5 & Veraltet & Krankheit & Niedriger IQ & Kognitive Einschränkungen \\
\hline 6 & Psychose & Schizophrenie & Kaum geistige Fähigkeiten & Lernschwierigkeiten \\
\hline 7 & Missbrauch/Vergewaltigung & Therapie & Wertend/abfällig & IQ unter 70 \\
\hline 8 & Trauma & Leid/Leidensdruck & Unsinn & $\begin{array}{l}\text { Probleme mit Lesen/Schreiben/ } \\
\text { Rechnen }\end{array}$ \\
\hline 9 & Unmensch/Monster & Psychose & Quatsch/Blödsinn & Einschränkungen im Alltag \\
\hline 10 & Rechtsbegriff & Emotional instabil & Verrückt & Folgen nicht abschätzen können \\
\hline
\end{tabular}


Tab. 4 Ergebnisse einer zweifaktoriellen ANOVA für SASA/SASS

\begin{tabular}{lllll}
\hline & & $\mathrm{F}(1,204)$ & $p$ & $\eta_{\mathrm{p}}^{2}$ \\
\hline Schuldfähigkeit & Haupteffekt Begriff & 1,05 & 0,307 & 0,005 \\
& Haupteffekt Vorwissen & 3,75 & 0,054 & 0,018 \\
& Interaktionseffekt & 0,39 & 0,533 & 0,002 \\
Einsichtsfähigkeit & Haupteffekt Begriff & 1,55 & 0,215 & 0,277 \\
& Haupteffekt Vorwissen & 1,19 & 0,866 & 0,008 \\
& Interaktionseffekt & 0,029 & 0,518 & 0,000 \\
Gefährlichkeit & Haupteffekt Begriff & 0,419 & $<0,001$ & 0,002 \\
& Haupteffekt Vorwissen & 15,71 & 0,757 & 0,072 \\
& Interaktionseffekt & 0,096 & 000 & 0 \\
\hline
\end{tabular}

Anmerkung. $F=$ F-Wert, $p=$ Signifikanz, $\eta_{p}{ }^{2}=$ partielles Eta ${ }^{2}$

Tab. 5 Mittlere Bewertungen der AV bei SASA/SASS

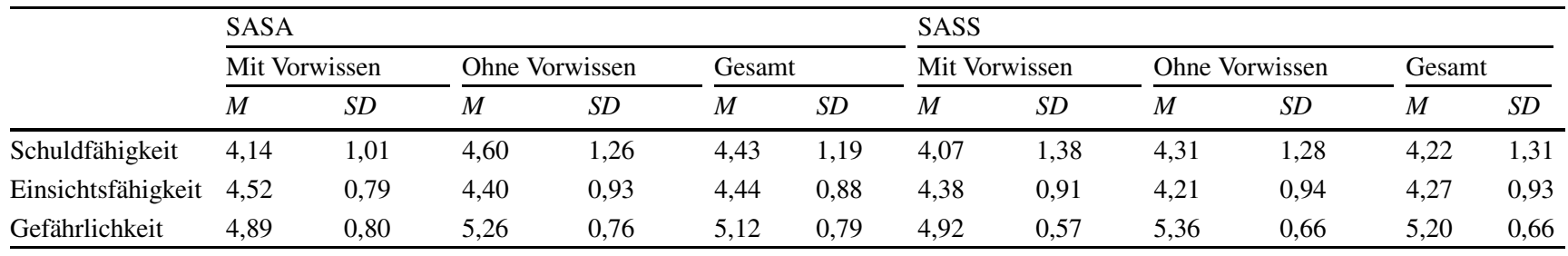

Anmerkung. Verwendet wurde eine 7-Punkte-Ratingskala, auf der hohe Werte für eine hohe Einschätzung sprechen

Tab. 6 Ergebnisse einer rmANOVA für Schwachsinn/Intelligenzminderung

\begin{tabular}{lllll}
\hline & & $F(1,206)$ & $p$ & $\eta_{p}{ }^{2}$ \\
\hline Schuldfähigkeit & Haupteffekt Begriff & 0,101 & 0,751 & $<0,001$ \\
& Haupteffekt Vorwissen & 17,39 & 0,000 & 0,078 \\
& Interaktionseffekt & 1,15 & 0,285 & 0,844 \\
Einsichtsfähigkeit & Haupteffekt Begriff & 0,039 & 0,516 & 0,000 \\
& Haupteffekt Vorwissen & 0,424 & 0,105 & 0,002 \\
& Interaktionseffekt & 2,65 & 0,255 & 0,013 \\
& Haupteffekt Begriff & 1,30 & 0,088 & 0,006 \\
& Haupteffekt Vorwissen & 2,94 & 0,417 & 0,003 \\
\hline
\end{tabular}

Anmerkungen. $F=\mathrm{F}-$ Wert, $p=$ Signifikanz, $\eta_{p}^{2}=$ partielles $\mathrm{Eta}^{2}$

Tab. 7 Mittlere Bewertungen der AV bei Schwachsinn/Intelligenzminderung

\begin{tabular}{|c|c|c|c|c|c|c|c|c|c|c|c|c|}
\hline & \multicolumn{6}{|c|}{ Schwachsinn } & \multicolumn{6}{|c|}{ Intelligenzminderung } \\
\hline & \multicolumn{2}{|c|}{ Mit Vorwissen } & \multicolumn{2}{|c|}{ Ohne Vorwissen } & \multicolumn{2}{|c|}{ Gesamt } & \multicolumn{2}{|c|}{ Mit Vorwissen } & \multicolumn{2}{|c|}{ Ohne Vorwissen } & \multicolumn{2}{|c|}{ Gesamt } \\
\hline & $M$ & $S D$ & $M$ & $S D$ & $M$ & $S D$ & $M$ & $S D$ & $\bar{M}$ & $S D$ & $M$ & $S D$ \\
\hline Schuldfähigkeit & 2,47 & 1,31 & 3,30 & 1,62 & 2,99 & 1,56 & 2,64 & 1,18 & 3,20 & 1,45 & 3,0 & 1,40 \\
\hline Einsichtsfähigkeit & 2,35 & 0,96 & 2,57 & 1,21 & 2,50 & 1,14 & 2,51 & 0,95 & 2,45 & 1,01 & 2,47 & 0,99 \\
\hline Gefährlichkeit & 5,44 & 0,85 & 5,57 & 0,93 & 5,52 & 0,90 & 5,30 & 0,76 & 5,55 & 0,93 & 5,46 & 0,89 \\
\hline
\end{tabular}

Anmerkung. Verwendet wurde eine 7-Punkte-Ratingskala, auf der hohe Werte für eine hohe Einschätzung sprechen

Die Begriffe Schwachsinn bzw. Intelligenzminderung wurden als Innersubjektfaktor manipuliert, sodass Probanden jeden Begriff einmal präsentiert bekamen. Im Folgenden wird für die jeweiligen Skalen verglichen, ob die Begriffe unterschiedliche Personenwahrnehmung nach sich zogen - abhängig vom Vorwissen der Probanden.
Auch in diesem Falle ergab die Verwendung der Begriffe Schwachsinn oder Intelligenzminderung entgegen der Hypothese weder signifikante Unterschiede in der Bewertung der Schuldfähigkeit noch in Einsichtsfähigkeit oder Gefährlichkeit (Tab. 7 für deskriptive Werte). Thematisches Vorwissen wirkte sich hier allerdings signifikant auf die Bewertungen der Schuldfähigkeit aus, jedoch nicht 
auf die Bewertung von Einsichtsfähigkeit oder Gefährlichkeit. Keine Effekte des Eingangsmerkmals Schwachsinn/ Intelligenzminderung wurden signifikant durch Vorwissen moderiert. Für die zusätzlichen - explorativ erhobenen Variablen Sympathie, Therapieempfänglichkeit, Rückfallrisiko, Abweichung von den geltenden Normen, Vollbesitz geistiger Kräfte sowie Impulssteuerung ergaben sich für dieses Eingangsmerkmal keine Effekte (Zusatzmaterial online: Tabelle A6).

\section{Diskussion}

Die schon seit Langem geforderte Reformierung der Begriffe Schwachsinn und Abartigkeit in §20 StGB, die 2019 vom BMJV in einem Entwurf zur Gesetzesänderung vorgelegt wurde, bildete den Ausgangspunkt für die vorliegende Studie. Ziel war es, näher zu beleuchten, für wie stigmatisierend die Begriffe tatsächlich gehalten werden, und in welchem Ausmaß sich die stigmatisierende Wirkung der Begriffe im Sinne des Labeling-Effekts auf die Personenwahrnehmung auswirkt. In den Ergebnissen zeigt sich deutlich, dass sowohl Schwachsinn als auch die schwere andere seelische Abartigkeit für stigmatisierender als ihre modernisierten Alternativen gehalten werden und dass sie als abwertender, negativer konnotiert und weniger juristisch angemessen empfunden werden. Auch in den Ergebnissen der qualitativen Analyse ist sichtbar, dass mit den alten Begriffen negativere Assoziationen verbunden werden.

Dennoch findet sich in unseren Daten keine starke Evidenz dafür, dass die Begriffe tatsächlich zu einer Einfärbung der Personenwahrnehmung führen (lediglich für die explorativ erfassten Einschätzungen finden sich im Falle des alten Begriffs der seelischen Abartigkeit eine höhere Einschätzung für den Vollbesitz geistiger Kräfte und geringere Einschätzungen der Impulssteuerung als für den novellierten Begriff der seelischen Störung). Für juristische und psychologische Experten ließe sich das damit erklären, dass sie die Begriffe als veraltet einordnen und ihre negative Wirkung neutralisieren können. Jedoch zeigten sich in unserer Studie auch für Laien, für Menschen also, die den Gebrauch dieser Terminologie weder gewohnt sind noch ihn kontextualisierend „entschärfen“ können, keinerlei Auswirkungen auf die eingeschätzte Schuld- und Einsichtsfähigkeit sowie Gefährlichkeit. Tatsächlich fand sich für keine der untersuchten Variablen eine Moderation in dem Sinne, dass die Begriffe eine unterschiedliche Wirkung je nach Vorwissen hatten (Hypothese 4). Theoretisch weniger relevante Haupteffekte geben einen Hinweis darauf, dass Befragte mit Vorwissen zuweilen weniger Schuldfähigkeit (für Intelligenzminderung) und weniger Gefährlichkeit (für seelische Störung) attestieren, dies jedoch unabhängig von der Terminologie der Eingangsmerkmale (Hypothesen 1-3).
Fraglich ist, weshalb die Begriffe so deutlich als stigmatisierend empfunden werden und dennoch keine Auswirkung auf die Personenwahrnehmung zu haben scheinen. Es kann ausgeschlossen werden, dass die in den Vignetten enthaltenen Informationen u. a. über Tat und Biografie der Täter das durch die Gutachter bestätigte Eingangsmerkmal überlagern. Da die Vignetten jeweils zufällig mit den Begriffen gepaart waren, sollten sich Effekte der genauen Beschreibung herausmitteln. Es ist jedoch möglich, dass die Begrifflichkeit in den Fallvignetten nicht ausreichend hervorstach, um die Personenwahrnehmung zu beeinflussen. Dies könnte einerseits als Schwäche des Designs interpretiert werden, da nicht ganz sichergestellt werden kann, ob Probanden die genauen Begriffe überhaupt ausreichend wahrgenommen haben. Andererseits ließe sich dies auch auf die Realität übertragen. Auch vor Gericht ist es durchaus möglich, dass das nur kurz erwähnte Eingangsmerkmal nur wenig Einfluss auf die Einschätzung der Beurteilten haben. Da dort ebenfalls eine Fülle von Informationen zu den Beurteilten vorliegt und die Entscheidungen vor Gericht nur zu einem Bruchteil auf den Eingangsmerkmalen basieren, ist es durchaus plausibel, dass sich die Begrifflichkeiten nur wenig auswirken. Eine weitere Erklärung für die mangelnde Auswirkung der Begriffe könnte die Tatsache sein, dass sich Probanden darüber im Klaren sein könnten, dass juristische Formulierungen häufig nicht umgangssprachlichen Formulierungen entsprechen. So ist es möglich, dass Probanden die Formulierungen zwar (negativ) auffallen, sie es aber im Rahmen der Personenbeurteilung ausblenden.

Interessanterweise gab es deutlich weniger Unterschiede durch juristisches bzw. rechtspsychologisches Vorwissen, als zunächst erwartet wurde. Es wurde von 77 der Probanden berichtet, solch thematisches Vorwissen zu haben. Die Forschung weist darauf hin, dass Experten unterschiedlich urteilen. Experten (i.d.F. Personen mit Vorwissen) und Laien unterscheiden sich dabei bezüglich ihres Wissens u.a. in ihren mentalen Repräsentationen von bestimmten Konzepten. Abhängig davon, welche Informationen zu einem Wissensgebiet oder einem Konzept verfügbar sind, werden spezifische oder weniger spezifische Vorstellungen abgerufen (Köhler 2009). Dieses domänenspezifische Wissen zu einem Wissensgebiet ist dabei bei Experten strukturierter als das Wissen von Laien. Fachbegriffe dienen bei Experten als Grundlage für kategoriale Wahrnehmung und haben demnach nicht nur eine rein kommunikative Funktion (Bromme und Rambow 2001). Trotz dieser Forschungslage unterstützen die Ergebnisse dieser Studie diese Unterschiede zwischen Laien und Experten nicht. Dies kann u. a. dadurch begründet sein, dass sich Vorwissen fast ausschließlich auf psychologisches Vorwissen beschränkte und somit wenig juristisches Vorwissen vorhanden war. Zusätzlich waren die Gruppen in ihrer Größe nicht ausgeglichen. 


\section{Limitationen und Ausblick}

Trotz des vielseitigen Studiendesigns gibt es verschiedene Limitationen, die beachtet werden müssen. Obwohl die Fallvignetten an Gutachten orientiert waren und somit so realitätsgetreu wie möglich gestaltet wurden, kann die Studie einer Situation vor Gericht nicht vollständig gerecht werden. So war die Studiensituation einer Kurzbeschreibung der Personen und ihrer Fälle nicht mit der Fülle an Informationen in Begutachtungen im Rahmen gerichtlicher Prozesse zu vergleichen. Ebenfalls waren der Anteil der Rechtswissenschaftler in der vorliegenden Stichprobe sehr gering, sodass hier kein Vergleich zu Psychologen und Laien gezogen werden konnte. Es handelt sich bei unserer Studie zusätzlich um eine hauptsächlich studentische Stichprobe. Zukünftige Studien sollten die Studiensituation stärker in einen gerichtlichen Rahmen bringen. Zuletzt sollte angemerkt werden, dass es durch den entstandenen Fehler bei der Randomisierung nicht für beide Eingangsmerkmale möglich war, einen Zwischensubjektvergleich zu berechnen.

Die vorliegende Studie zeigt deutlich, dass die Terminologie des $\$ 20 \mathrm{StGB}$ als stigmatisierend wahrgenommen wird. Dennoch wirkt sich diese Stigmatisierung im Sinne des Labeling-Effektes nicht auf die Personenwahrnehmung aus und scheint so faire Verfahren nicht zu gefährden. Dennoch wird deutlich, dass eine Modernisierung der Terminologie angebracht und sinnvoll ist. Sollte der Gesetzesentwurf des BMJV angenommen werden, wäre nach der Einführung der modernisierten Begriffe eine empirische Überprüfung des tatsächlichen Einflusses vor Gericht denkbar.

Funding Open Access funding enabled and organized by Projekt DEAL.

Interessenkonflikt D. Rösch, S. Ruckelshaußen, J. Kirsch, S. Gerhards, L.A. Sroka und R. Imhoff geben an, dass kein Interessenkonflikt besteht.

Open Access Dieser Artikel wird unter der Creative Commons Namensnennung 4.0 International Lizenz veröffentlicht, welche die Nutzung, Vervielfältigung, Bearbeitung, Verbreitung und Wiedergabe in jeglichem Medium und Format erlaubt, sofern Sie den/die ursprünglichen Autor(en) und die Quelle ordnungsgemäß nennen, einen Link zur Creative Commons Lizenz beifügen und angeben, ob Änderungen vorgenommen wurden.

Die in diesem Artikel enthaltenen Bilder und sonstiges Drittmaterial unterliegen ebenfalls der genannten Creative Commons Lizenz, sofern sich aus der Abbildungslegende nichts anderes ergibt. Sofern das betreffende Material nicht unter der genannten Creative Commons Lizenz steht und die betreffende Handlung nicht nach gesetzlichen Vorschriften erlaubt ist, ist für die oben aufgeführten Weiterverwendungen des Materials die Einwilligung des jeweiligen Rechteinhabers einzuholen.

Weitere Details zur Lizenz entnehmen Sie bitte der Lizenzinformation auf http://creativecommons.org/licenses/by/4.0/deed.de.

\section{Literatur}

Bromme R, Rambow R (2001) Experten-Laien-Kommunikation als Gegenstand der Expertiseforschung: Für eine Erweiterung des psychologischen Bildes vom Experten. In: Silbereisen RK, Reitzle M (Hrsg) Psychologie 2000. Bericht über den 42. Kongress der Deutschen Gesellschaft für Psychologie in Jena 2000. Pabst Science Publishers, Lengerich, S 541-550

Bundesministerium für Justiz und Verbraucherschutz (2019) Gesetz zur Änderung des Strafgesetzbuches - Modernisierung des Schriftenbegriffs und anderer Begriffe sowie Erweiterung der Strafbarkeit nach den $\S \S 86,86 a, 111$ und 130 des Strafgesetzbuches bei Handlungen im Ausland (Referentenentwurf). https://www.bmjv.de/SharedDocs/Gesetzgebungsverfahren/DE/ Schriftenbegriff.html. Zugegriffen: 29. Nov. 2020

Bundesministerium für Justiz und Verbraucherschutz (2020) Gesetz zur Änderung des Strafgesetzbuches - Modernisierung des Schriftenbegriffs und anderer Begriffe sowie Erweiterung der Strafbarkeit nach den $\S \S 86,86 a, 111$ und 130 des Strafgesetzbuches bei Handlungen im Ausland (Regierungsentwurf). https://www.bmjv.de/SharedDocs/Gesetzgebungsverfahren/DE/ Schriftenbegriff.html. Zugegriffen: 5. Sept. 2020

Deutsche Heilpädagogische Gesellschaft e.V. (2013) Menschen mit geistiger Behinderung im Maßregelvollzug. DHG, Jülich. http:// dhg-kontakt.de/wp-content/uploads/2015/12/DHG-Schrift-18. pdf. Zugegriffen: 14. Mai 2020

Feldman DB, Crandall CS (2007) Dimensions of mental illness stigma: What about mental illness causes social rejection? J Soc Clin Psychol 26(2):137-154

Imhoff R (2015) Punitive attitudes against pedophiles or persons with sexual interest in children: does the label matter? Arch Sex Behav $44: 35-44$

Imhoff R (2016) Zeroing in on the effect of the schizophrenia label on stigmatizing attitudes: a large scale study. SCHBUL 42:456-463

Janßen M (1994) Unter der Last der Normen: Über die Schwierigkeiten, normal und anormal im strafrechtlichen Feld zu unterscheiden (Magisterarbeit). Gottfried Wilhelm Leibnis Universität, Hannover. https://doi.org/10.15488/254

Köhler S (2009) Die Auswirkung des Wissensungleichgewichtes zwischen Experten und Laien auf die Bewertung von Gebäuden in Sichtbetonbauweise (Forschungsbericht). Dresden. http:// www.architekturpsychologie-dresden.de/ddarbeiten/koehler_ fov_sichtbeton.pdf. Zugegriffen: 6. Sept. 2020

Krümpelmann J (1976) Die Neugestaltung der Vorschriften über die Schuldfähigkeit durch das Zweite Strafrechtsreformgesetz vom 4.Juli 1969. Z Gesamt Strafrechtswiss 88(1):6-39

Link BG, Cullen FT, Struening E, Shrout PE, Dohrenwend BP (1989) A modified labeling theory approach to mental disorders: an empirical assessment. Am Sociol Rev 54:400-423

Meyer J-E (1976) Psychiatrische Diagnosen und ihre Bedeutung für die Schuldfähigkeit im Sinne der $\S \S 20 / 21$. Z Gesamt Strafrechtswiss $88(1): 46-56$

Nedopil N (2007) Forensische Psychiatrie. Thieme, Leipzig

Rechtsanwaltskanzlei Strate und Ventzke (2020a) HRRS 2004 Nr. 415 (LG Stuttgart) hrr-strafrecht.de. https://www.hrr-strafrecht.de/hrr/ 1/03/1-346-03.php3?referer=db. Zugegriffen: 14. Mai 2020

Rechtsanwaltskanzlei Strate und Ventzke (2020b)HRRS 2011 Nr. 196 (LG Kiel) hrr-strafrecht.de. https://www.hrr-strafrecht.de/hrr/5/ 10/5-240-10.php?referer=db. Zugegriffen: 14. Mai 2020

Rechtsanwaltskanzlei Strate und Ventzke (2020c)HRRS 2018 Nr. 832 (LG Mühlhausen) hrr-strafrecht.de. https://www.hrr-strafrecht. de/hrr/2/18/2-112-18.php. Zugegriffen: 14. Mai 2020

Schiemann A (2019) Weg mit dem Schwachsinn: zur längst überfälligen Ersetzung der Begriffe „Schwachsinn“" und „Abartigkeit“ in $\S 20$ StGB und der verpassten Chance einer umfassenden Reform der Schuldfähigkeitsfeststellung. KriPoZ 6:338-346 
Schneider K (2007) Klinische Systematik und Krankheitsbegriff. In: Schneider K (Hrsg) Klinische Psychopathologie, 15. Aufl. Thieme, Leipzig

Schneider F, Frister H, Olzen D (2020) Begutachtung psychischer Störungen. Springer, Berlin

Schweizer MD (2005) Kognitive Täuschungen vor Gericht: eine empirische Studie. Dissertation. University of Zürich, Rechtswissenschaftliche Fakultät, Zürich. https://doi.org/10.5167/uzh-165152
Theune W (2002) Die Beurteilung der schweren anderen seelischen Abartigkeit in der Rechtsprechung und ihre Vereinbarkeit mit dem Schuldprinzip. Z Gesamt Strafrechtswiss 114(2):300-318

Wegener B (1989) Seelische Abartigkeit ( 20 Strafgesetzbuch): Ein schwieriger Begriff der forensischen Psychiatrie / Psychologie. Krit Justiz 22(3):316-328 